\title{
DESENHO E IMPLANTAÇÃO DE PROCESSOS DE GESTÃO PARA AUTOMAÇÃO INDUSTRIAL*
}

\author{
Vinicius Andres Strey ${ }^{1}$ \\ Jorge Eustáquio de Campos Filho ${ }^{2}$ \\ Márcio Aurélio dos Santos ${ }^{3}$ \\ Cristiano Coelho Ferreira ${ }^{4}$ \\ Vinícius Vilela Wiermann ${ }^{5}$ \\ Marcelo Antônio Quintino de Paula
}

\section{Resumo}

A estruturação organizacional da rotina operacional em processos de gestão já está consolidada no ambiente de Tecnologia da Informação (TI). O ambiente de Tecnologia de Automação (TA) compartilha características semelhantes à TI, porém implantar processos de gestão no ambiente de TA de uma empresa já existente demanda uma série de ações para garantir que este sistema de gestão está alinhado aos objetivos da organização, que este sistema de gestão está sendo realmente utilizado e sustentável e, principalmente, que este sistema de gestão é aderente aos requisitos da rotina da TA. Os principais retornos sobre o investimento neste tipo de projeto são em termos de organização das atividades e produtividade de pessoal, de disponibilidade dos ativos pela resposta planejada e rápida a falhas e de visibilidade da TA dentro da organização. Este trabalho define as diretrizes para o desenho e implantação de processos de gestão. Estas diretrizes foram empregadas em projetos de Metais e Mineração, como no caso específico do Departamento de Automação e Instrumentação da Samarco Mineração.

Palavras-chave: Convergência TI/TA; Tecnologia de automação; Governança.

\section{DESIGN AND IMPLEMENTATION OF MANAGEMENT PROCESSES FOR INDUSTRIAL AUTOMATION}

\section{Abstract}

The organizational structure of the operational routine in management processes is already established in the Information Technology (IT) environment. The Operational Technology (OT) environment shares similar characteristics to IT, however, to implement management processes in the OT environment of an existing company demands a lot of actions to ensure this management system is aligned with the corporative objectives, this management system is actually being used and sustainable and mainly this management system is adherent to the OT's routine requirements. The main returns on investment in this kind of project are in terms of organization of the activities and staff productivity, asset availability due to the planned and fast response to failures and the OT visibility inside the organization. This paper gives the guidelines for design and implementation of management processes. These guidelines were used within Metals and Mining projects, such as in the specific case of the Automation and Instrumentation Department of Samarco Mineração.

Keywords: IT/OT convergence; Operational technology; Governance.

1 Graduado em Engenharia de Controle e Automação, PMP, CRISC, Engenheiro de Sistemas e Soluções Sênior, TI Industrial, Chemtech Serv. de Eng. e Soft., Belo Horizonte, MG, Brasil.

2 Graduado em Engenharia Química, Pós-Graduado em Automação Industrial, Líder de Projetos, TI Industrial, Chemtech Serv. de Eng. e Soft., Belo Horizonte, MG, Brasil

3 Graduado em Computação, Pós-Graduado em Automação Industrial, Analista de Automação Sênior, Departamento de Automação e Instrumentação, Samarco Mineração, Mariana, MG, Brasil

4 Graduado em Engenharia Eletrônica e de Telecomunicações, Engenheiro de Automação Pleno, Departamento de Automação e Instrumentação, Samarco Mineração, Mariana, MG, Brasil

5 Graduado em Engenharia Elétrica (UFMG), Pós-Graduado em Automação Industrial, Engenheiro Especialista, Departamento de Automação e Instrumentação, Samarco Mineração, Mariana, MG, Brasil

6 Graduado em Ciências da Computação, Analista de Automação, Departamento de Automação e Instrumentação, Samarco Mineração, Ubu, MG, Brasil 


\section{INTRODUÇÃO}

Tradicionalmente, o fluxo de informação dentro de grandes empresas está dividido em ambiente de Tecnologia de Informação (TI) e ambiente de Tecnologia de Automação (TA). A TI e a TA compartilham muitas semelhanças, mas possuem importantes diferenças sobre o propósito, arquitetura, propriedade da informação, conectividade e os sistemas gerenciados [1].

A TI, por atuar em níveis superiores da informação e, consequentemente, mais próxima da Alta Direção das empresas, estruturou-se há mais tempo como um parceiro estratégico para o negócio. Isto se reflete na estrutura hierárquica da TI, que conta com o $\mathrm{ClO}$, uma figura corporativa com grande poder político nas organizações. A TA, por estar próxima ao "chão de fábrica" e por compartilhar com outros grupos a manutenção da operação, se distanciou cada vez mais da estratégia corporativa preocupando-se, cada vez mais, com o gerenciamento de sua rotina operacional.

A TA, em sua busca por aumentar a sua agilidade $e$ atender às demandas operacionais, faz uso cada vez mais extensivo das tecnologias da TI, tais como switches, sistemas operacionais, protocolos de rede e, até mesmo, virtualização. Esta aproximação, somada ao fato de que a qualidade e a disponibilidade dos serviços de automação impactam diretamente o resultado e o fluxo de caixa das empresas, acabou fazendo com que a Alta Direção e a própria TI começassem a enxergar a TA como um parceiro estratégico. Esta redução do gap tecnológico entre os dois ambientes deu origem ao processo de Convergência TI/TA [2]. Entretanto, desprezando-se o fator tecnológico supracitado, ainda há uma distância considerável entre TI e TA nos outros fatores organizacionais.

\section{FATORES ORGANIZACIONAIS}

Além da supracitada Tecnologia, os outros fatores organizacionais são os Processos de Gestão e as Pessoas. Como já dito, tecnologicamente a TA e a TI se aproximaram. Para entender esta preferência da TA pela parte tecnológica, é necessário fazer um parêntesis quanto aos aspectos de segurança da informação, que são disponibilidade, integridade e confidencialidade.

A TA sempre privilegiou a disponibilidade dos seus serviços, enquanto a TI atua mais sobre a confidencialidade. A visão tradicional da TA é resolver seus problemas de disponibilidade com estratégias de alta tolerância a falhas, como equipamentos de alto MTBF e redundâncias de ativos e enlaces. Enquanto isso, na TI percebeu-se que para manter a confidencialidade não basta apenas a aquisição de firewalls ou de sistemas de prevenção de intrusão, mas sim é necessário investir em Processos de Gestão que definam papéis e responsabilidades corporativamente.

Desta forma, não são raros os casos em que a disponibilidade do processo produtivo fica comprometida devido a problemas em Processos de Gestão de TA. $80 \%$ dos problemas de disponibilidade do processo produtivo gerados pela TA surgem devido a problemas em Processos de Gestão ou Pessoas, enquanto apenas $20 \%$ derivam de Tecnologia [3].

Em um cenário de crise, a TA e as outras áreas operacionais sofrem pressões para redução de custo e manutenção da operação. A agregação de mais tecnologias para atender esta situação latente gera dois problemas: 
- Quanto mais tecnologias, mais serviços a equipe deve gerenciar. Desta forma, precisa-se de mais pessoas. Entretanto, como conciliar isto com pressões para redução de custo?

- Quanto mais tecnologias, mais custoso é o próximo salto tecnológico para obter o mesmo benefício.

Para a TA ser competitiva dentro da organização deve continuar a investir racionalmente em Tecnologia, entretanto precisa atuar proativamente também em Pessoas e Processos de Gestão. Este último, essencialmente, será o foco deste trabalho.

\section{SISTEMA DE GERENCIAMENTO DE SERVIÇOS DE AUTOMAÇÃO}

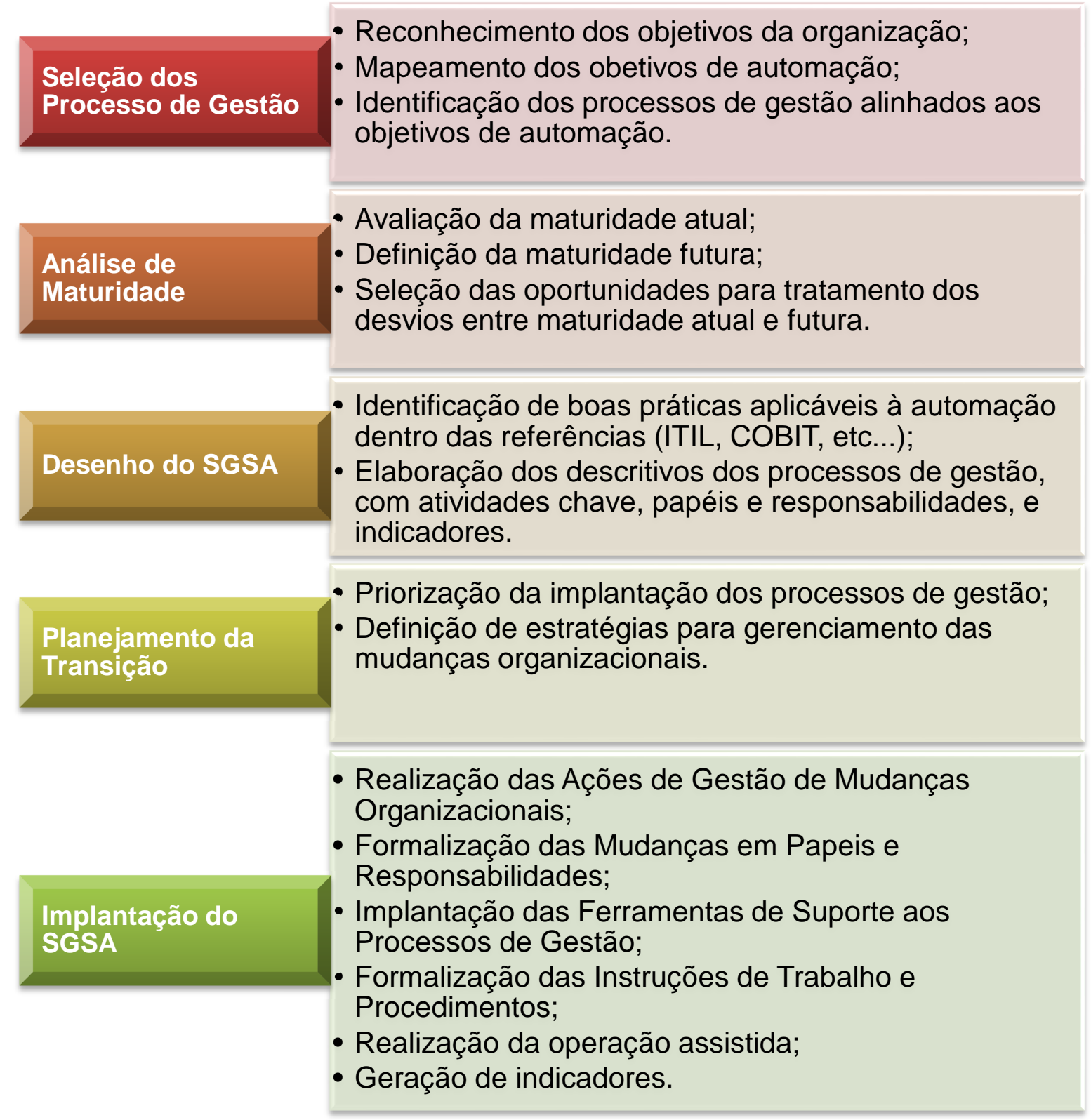

Figura 1. Modelo conceitual para implantação do SGSA

O Sistema de Gerenciamento de Serviços de Automação (SGSA) atua primordialmente sobre este fator organizacional dos Processos de Gestão. Esta iniciativa já é empregada no ambiente de $\mathrm{TI}$, dado que já há um conjunto de 
frameworks, normas e guias de boas práticas, como o COBIT [4] e a ITIL [5], respectivamente, que definem metodologias para a construção de um sistema de governança. Todos estes itens estão estruturados sobre o conceito de serviços.

Adaptando-se o conceito da ITIL v3 para serviços de TI, pode-se afirmar que um serviço de TA é um serviço provido para um ou mais clientes, que suporta os processos de negócios destes clientes, feito de uma combinação de pessoas, processos e tecnologia e, deve ser definido por acordos de nível de serviço.

Podem ser citados como exemplos de serviços de TA: Controle Regulatório, Controle Avançado, Sistema de Supervisão, Gerenciamento de Ativos de Instrumentação e Gerenciamento dos Controladores de Domínio.

A metodologia apresentada adiante, representada na Figura 1, define uma estrutura de atividades a serem conduzidas para que a consolidação de um SGSA.

\section{SELEÇÃO DOS PROCESSOS DE GESTÃO}

Inicialmente, deve ser realizada a seleção dos processos de gestão. O COBIT e a ITIL definem processos de gestão para o ambiente de TI, que podem ser adaptados para o ambiente de TA. Alguns dos processos de gestão do COBIT que possuem alto potencial de aplicabilidade para o ambiente de TA estão destacados na Figura 2.

\begin{tabular}{|c|c|c|c|}
\hline PO1 & Definir um Plano Estratégico de TA & DS1 & $\begin{array}{l}\text { Definir e Gerenciar Níveis de } \\
\text { Servicos }\end{array}$ \\
\hline PO2 & Definir a Arquitetura da Informação & DS2 & Gerenciar Serviços de Terceiros \\
\hline $\mathrm{PO3}$ & $\begin{array}{l}\text { Determinar o Direcionamento } \\
\text { Tecnológico }\end{array}$ & DS3 & Gerenciar Capacidade e Desempenho \\
\hline $\mathrm{PO} 4$ & $\begin{array}{l}\text { Definir os Processos, Organização e os } \\
\text { Relacionamentos de TA }\end{array}$ & DS4 & $\begin{array}{l}\text { Assegurar Continuidade de } \\
\text { Servicos }\end{array}$ \\
\hline PO5 & Gerenciar o Investimento de TA & DS5 & Assegurar a Segurança dos Serviços \\
\hline PO6 & $\begin{array}{l}\text { Comunicar as Diretrizes e Expectativas } \\
\text { da Diretoria }\end{array}$ & DS6 & Identificar e Alocar Custos \\
\hline $\mathrm{PO7}$ & Gerenciar os Recursos Humanos de TA & DS7 & Educar e Treinar os Usuários \\
\hline $\mathrm{PO}$ & Gerenciar a Qualidade & DS8 & $\begin{array}{l}\text { Gerenciar a Central de Servico e os } \\
\text { Incidentes }\end{array}$ \\
\hline PO9 & Avaliar e Gerenciar os Riscos de TA & DS9 & Gerenciar a Configuração \\
\hline PO10 & Gerenciar Projetos & DS10 & Gerenciar os Problemas \\
\hline Al1 & Identificar Soluções Automatizadas & DS11 & Gerenciar os Dados \\
\hline $\mathrm{Al} 2$ & Adquirir e Manter Software Aplicativo & DS12 & Gerenciar o Ambiente Físico \\
\hline $\mathrm{Al}$ & $\begin{array}{l}\text { Adquirir e Manter Infraestrutura de } \\
\text { Tecnologia }\end{array}$ & DS13 & Gerenciar as Operações \\
\hline Al4 & Habilitar Operação e Uso & ME1 & Monitorar e Avaliar o Desempenho \\
\hline Al5 & Adquirir Recursos de TA & ME2 & $\begin{array}{l}\text { Monitorar e Avaliar os Controles } \\
\text { Internos }\end{array}$ \\
\hline Al6 & Gerenciar Mudanças & ME3 & $\begin{array}{l}\text { Assegurar a Conformidade com } \\
\text { Requisitos Externos }\end{array}$ \\
\hline Al7 & $\begin{array}{l}\text { Mstalar e Homologar Soluçōes e } \\
\text { Mudanças }\end{array}$ & ME4 & Prover a Governança de TA \\
\hline
\end{tabular}

Figura 2. Processos de gestão do COBIT com alto potencial de aplicabilidade para a TA 
A seleção final dos processos de gestão a serem implantados deve considerar os Objetivos de TA. O COBIT 4.1, no Apêndice I, fornece um guia para o mapeamento entre os Objetivos de TA e os Processos de Gestão. Por exemplo, caso um dos Objetivos de TA seja "Assegurar a conformidade de TA com leis, regulamentos e contratos", corrobora-se a implantação do processo de gestão "Monitorar e avaliar os controles internos".

Fato é que muitas gerências, departamentos e áreas de TA não possuem seus Objetivos de TA mapeados. Estes objetivos devem obrigatoriamente derivados dos Objetivos do Negócio, que surgem do Planejamento Estratégico da Organização.

Os fatores críticos de sucesso no alinhamento entre os Objetivos de TA e os Objetivos do Negócio são o envolvimento da Alta Direção nos negócios da TA e a participação da TA no Planejamento Estratégico da Organização. Por sua vez, os principais fatores inibidores do alinhamento dos objetivos são a dificuldade na comunicação entre o pessoal de negócios e de TA e a falta de prioridade na TA pela organização, do que deriva o desinteresse da Alta Direção sobre esta área da empresa [6].

A realização do mapeamento entre Objetivos de Negócios e Objetivos da TA garante que a TA estará alinhada ao negócio na execução das suas iniciativas internas.

\section{AVALIAÇÃO DE MATURIDADE}

Após a seleção dos processos de gestão, procede-se com a avaliação de maturidade. O objetivo da análise de maturidade é determinar o desvio (gap) entre a maturidade em processos de gestão atual e a maturidade em processos de gestão futura. A maturidade de processos de gestão futura define um estágio desejado em algum ponto do futuro, tradicionalmente após a implantação do SGSA.

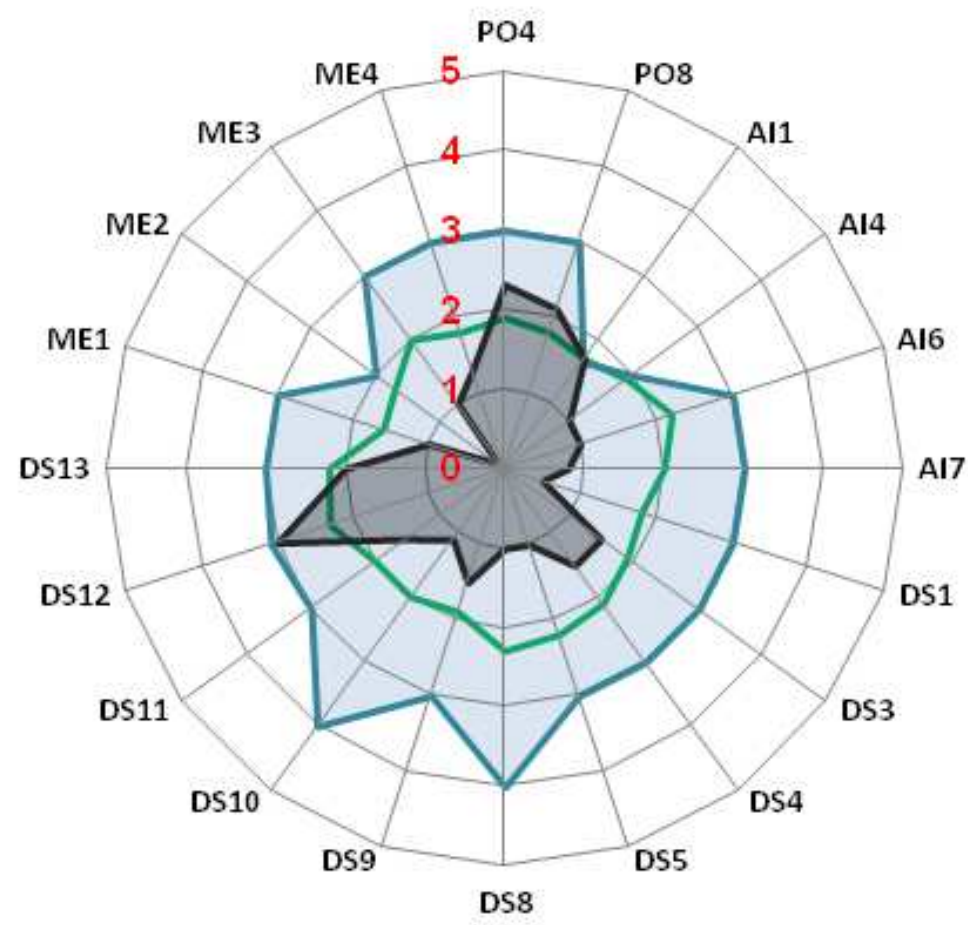

$\square$ Benchmark

$\square$ Mat. Futura

Mat. Atual

Figura 3. Exemplo de representação gráfica da análise de maturidade 
Não há impedimento para que seja realizada a avaliação de maturidade de todos os processos e em seguida a seleção dos mesmos. Entretanto, no COBIT 4.1, há 34 processos de gestão. A avaliação de maturidade de todos estes processos pode levar a um trabalho excessivo e com pouco valor agregado globalmente.

A avaliação de maturidade no SGSA tem por objetivo determinar os desvios (gaps). No COBIT 4.1, cada processo de gestão pode estar em seis níveis de maturidade, a saber: Inexistente (0), Inicial (1), Repetível (2), Processo Definido (3), Gerenciado e Mensurável (4) e Otimizado (5).

$A$ avaliação de maturidade deve ser realizada sobre a aderência às assertivas que definem cada um destes níveis. A ISACA, associação que mantém o COBIT, disponibiliza toolkit para análise de maturidade de processos de gestão para as versões mais recentes do framework, como o COBIT 4.1 e o COBIT 5.

A Figura 3 representa um exemplo do resultado final da análise de maturidade, na qual cada vértice do gráfico de radar representa um processo de gestão. A definição da maturidade futura não é um processo trivial e não deve representar apenas uma intenção. Pelo contrário, a maturidade futura deve refletir o planejamento estratégico da organização e, especialmente os objetivos da TA.

\section{DESENHO DO SGSA}

O desenho do SGSA é realizado em função do tratamento dos desvios identificados na análise de maturidade. Esta etapa envolve a elaboração da documentação dos processos de gestão. Um processo de gestão contem, pelo menos, os seguintes itens:

- Atividades Chave: estas atividades definem o fluxo de tarefas que devem ser conduzidas para a realização de um processo de gestão. Em um processo de gestão de mudanças, por exemplo, as atividades estão estruturadas conforme o ciclo de vida das mudanças, como "Cadastrar mudança", "Classificar mudança", "Planejar mudança" e "Executar mudança". As atividades são sumarizadas em fluxogramas de processo de negócio utilizando, por exemplo, o BPMN;

- Papéis e Responsabilidades: cada atividade chave deve estar atrelada a responsáveis dentro da estrutura organizacional. Normalmente estrutura-se como uma Matriz RACl;

- Indicadores de Desempenho: para assegurar a sustentabilidade e melhoria contínua do processo de gestão são definidos certos indicadores, que devem ser monitorados.

Dentro do ambiente de TA, é comum que sejam desenvolvidos procedimentos operacionais e instruções de trabalho. Entretanto, estes documentos, em regra, são desconexos e definem apenas como deve ser desempenhada certa atividade. Os processos de gestão, por sua vez, são documentos conexos, que definem "o que" fazer, "quem" faz e "como" monitorar. O fluxo adequado é que a partir das atividades dos processos de gestão, que estão alinhadas aos Objetivos da TA, é que serão elaborados os procedimentos operacionais e as instruções de trabalho.

O desenho do SGSA deve considerar, além dos resultados da análise de maturidade, as boas práticas descritas nos frameworks, normas e guias de boas práticas supracitados. Reforça-se que, para o ambiente de TA, estas referências devem ser adaptadas considerando as particularidades do ambiente, já que foram desenvolvidas inicialmente para TI. 
Além disso, o desenho do SGSA deve considerar as práticas em execução dentro da TA e da organização, a linguagem adotada internamente e as políticas e procedimentos operacionais já existentes, de forma a reduzir os riscos de implantação.

\section{PLANEJAMENTO DA TRANSIÇÃO}

A transição envolve a implantação dos processos de gestão gerados no SGSA. Para esta implantação deve ser definido um plano, de modo que haja tempo hábil para as pessoas envolvidas assimilarem a nova rotina proporcionada pelos novos processos de gestão. Tipicamente, esta implantação é feita em fases.

A priorização dos processos de gestão deve considerar especialmente as relações entre eles. Durante o Desenho do SGSA (seção 6), é observado um fluxo natural de informações entre os processos de forma que alguns deles são mais "fornecedores" de informação, enquanto outros são mais "consumidores". Os processos de gestão com menor dependência de outros processos ("fornecedores") possuem maior potencial para a implantação prioritária.

Para qualquer processo de gestão, entretanto, deve ser realizado um trabalho de gestão de mudanças organizacionais com suporte de alguma metodologia, por exemplo, ADKAR [7]. A gestão de mudanças organizacionais minimiza o risco de ocorrerem resistências internas, o que é mais forte na implantação de processos de gestão, pois envolvem alterações na rotina de trabalho da equipe.

\section{IMPLANTAÇÃO DOS PROCESSOS DE GESTÃO}

A implantação dos processos de gestão envolve basicamente a execução do planejamento da transição (seção 7) e realização de atividades adicionais para garantir a sustentabilidade da solução. A implantação envolve tipicamente:

- Realizar as Ações de Gestão de Mudanças Organizacionais: o planejamento realizado durante o Planejamento da Transição (seção 7) deve ser refinado considerando a situação real de implantação e executado;

- Formalizar as Mudanças em Papeis e Responsabilidades: as atividades dos processos de gestão e as matrizes RACl podem definir alterações nos papeis e responsabilidades organizacionais. Estes rearranjos organizacionais são necessários para contemplar os novos processos de gestão;

- Implantar as Ferramentas de Suporte aos Processos de Gestão: ver seção 9;

- Formalizar Instruções de Trabalho e Procedimentos: conforme já descrito na seção 6, o conteúdo dos processos de gestão é sucinto e assertivo. Eventualmente é necessário desenvolver instruções de trabalho e procedimentos detalhando como realizar algumas das atividades ou ainda formalizar roteiros de atividades técnicas realizadas pela equipe;

- Realizar a Operação Assistida: uma equipe de implantação, com experiência neste tipo de projeto, deve estar disponível para auxiliar as pessoas na realização das atividades de acordo com os novos processos de gestão;

- Gerar Indicadores: os indicadores são das ferramentas de sustentabilidade do SGSA mais importantes. Devem ser implantados de acordo com as definições nos processos de gestão. 


\section{FERRAMENTAS DE SUPORTE AOS PROCESSOS DE GESTÃO}

Conforme já mencionado, o fator tecnologia é amplamente explorado como uma das principais alternativas para se obter maior disponibilidade e eficiência no ambiente de automação. Desta forma, esta seção será voltada apenas para tecnologias relevantes para a implantação e manutenção dos processos de gestão. De uma forma geral, os sistemas mais demandados serão:

- Sistema de gerenciamento de chamados (tickets): alguns dos processos de gestão dependem destes sistemas para a rastreabilidade, como o "Al6 Gerenciar Mudanças" e o "DS8 - Gerenciar a Central de Serviços e os Incidentes". Exemplos de produtos no mercado: OTRS (Ver Figura 4), Atlassian JIRA, CA Service Desk, Omninet Omnitracker ITSM e Microsoft System Center;

- Sistema de gerenciamento de ativos: o monitoramento dos ativos, sejam ativos de rede ethernet, ativos da rede de controle ou ativos de instrumentação, é fonte importante para a identificação de incidentes e para a consolidação de inventário de ativos;

- Outros sistemas auxiliares, como sistemas de inventário de hardware e software, sistema de gerenciamento de backup e imagens, sistemas de gerenciamento de logs.

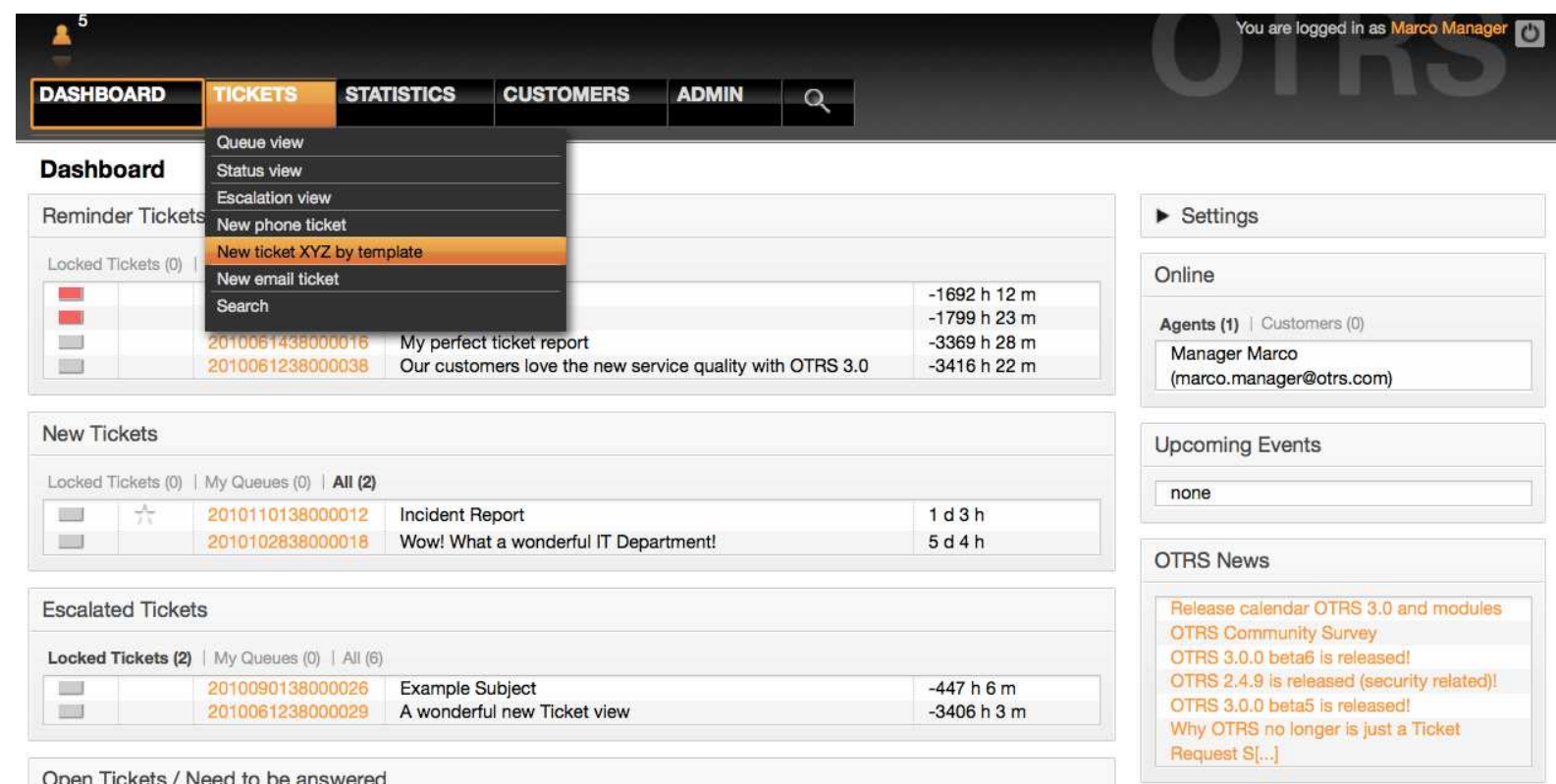

Open Tickets / Need to be answered

Figura 4. Tela exemplo de sistema para gerenciamento de chamados (OTRS) com boa aplicabilidade para ambiente de automação

É importante realizar um estudo técnico para seleção adequada das ferramentas, bem como uma análise de custo-benefício para as aquisições a serem feitas. Existem diversas soluções gratuitas ou de código aberto, mas que apresentam limitações de funcionalidades, de suporte ou mesmo de atualização.

Vale destacar que a instalação e configuração destas ferramentas podem demandar também modificações no ambiente de automação, como alterações de arquitetura, segurança, além da necessidade de hardware específico para hospedar os servidores. 


\section{CONCLUSÃo}

A estruturação formal de processos de gestão é algo inovador para a TA, até mesmo em nível global. Como não há frameworks e boas práticas de referência focadas em ambiente industrial, é necessário que sejam adaptadas referências de TI. Esta adaptação deve considerar as restrições do ambiente industrial.

Entretanto, o mercado de metais e mineração brasileiro é pioneiro neste tipo de projeto para a TA. Há sistemas de gestão (SGSA) sendo implantados em grandes empresas do setor. A Samarco Mineração segue na linha de frente, lançando mão deste modelo de gestão.

Já nas primeiras fases da implantação do SGSA na Samarco, foi possível perceber entre outros resultados, saltos em termos de organização da rotina e do ambiente industrial e da visibilidade da própria TA dentro da organização como uma área fundamental para o negócio. A organização da rotina já culminou com a detecção precoce de situações que afetariam a disponibilidade dos ativos e dos serviços de automação disponibilizados e, por consequência, do processo produtivo.

Estes resultados proporcionados pela implantação do SGSA são sentidos tanto pela equipe de TA, envolvida diretamente no SGSA, bem como pelos seus clientes internos (operação, manutenção, engenharia de processo, TI, entre outros).

Mais importante que os ganhos diretos, o desenvolvimento de processos de gestão junto com o desenvolvimento tecnológico e das pessoas permite um crescimento sustentável da TA e maior resiliência em momentos de crise.

\section{REFERÊNCIAS}

1 Steenstrup K. Integrate Operational Technology and IT for Business Success, Gartner. 2011.

2 Steenstrup K, Gerrard M. As IT and OT Merge, the Role of the Utility CIO Changes, Gartner. 2007.

3 Colville RJ, Spafford G. Configuration Management for Virtual e Cloud Infrastructures, Gartner, 2010.

4 ISACA (2007). CobiT 4.1. IT Governance Institute, Rolling Meadows/IL/USA.

5 OGC (2011). ITIL Lifecycle Publication Suite. The Stationery Office.

6 Laguna G, Oliveira, LR. Fatores Críticos de Sucesso para Alinhamento de Objetivos de Negócios e TI, III SEGeT, 2006.

7 Hiatt J. M. ADKAR: A Model for Change in Business, Government and our Community, Prosci, Loveland/CO/USA. 